\title{
Precuneus Brain Response Changes Differently During Human-Robot and Human-Human Dyadic Social Interaction
}

Nicolas Spatola ( $\sim$ Nicolas.spatola@iit.it)

Istituto Italiano di Tecnologia

Thierry Chaminade

Institut de Neurosciences de la Timone

\section{Research Article}

Keywords: Human-Robot Interaction, Functional Magnetic Resonance Imaging, Second-Person Neuroscience, Social cognition, Social Robotics

Posted Date: July 24th, 2021

DOI: https://doi.org/10.21203/rs.3.rs-691886/v1

License: (c) (i) This work is licensed under a Creative Commons Attribution 4.0 International License. Read Full License 


\section{Abstract}

Human-human and human-robot interaction are often compared with the overarching question of the differences in terms of cognitive processes engaged and what can explain these differences. However, research addressing this topic, especially in neuro-imagery, use extremely artificial interaction settings. Also, they neglect a crucial parameter of human social cognition: interaction is an adaptive (rather than fixed) process. Building upon the first fMRI paradigm requiring participants to interact online with both a human and a robot in a dyadic setting, we investigate the differences and changes of brain activity during the two type of interactions in a whole brain analysis. Our results show that, grounding on a common default level, the activity in specific neural regions associated with social cognition (e.g. Posterior Cingulate Cortex) increase in HHI while remaining stable in HRI. We discuss these results regarding the iterative process of deepening the social engagement facing humans but not robots.

\section{Significance Statement}

To what extent Human-Robot and Human-Human Interaction differ in terms of social engagement is a crucial question for societies aiming to introduce robots in various domains of human life. To address this issue, building upon an innovative fMRI paradigm, we investigate the changes of brain response during a direct interaction with a robot compared to a human. We show that despite a common level of social network activation at first, interacting with our fellows, we deepen our social engagement while, interacting with robots, we remain on a default level of social engagement. This study sheds light on the importance to consider the dynamic of social interaction with both humans and robots to understand how people engage and consider other agents.

\section{Introduction}

We, Humans, are intrinsically social beings. Throughout our evolution, social interactions have structured our behaviours, our brains and ultimately our environment ${ }^{1}$. Thanks to technology, our species continuously diversifies the way people engage with fellow humans, and more recently, with artificial agents such as social robots. More than providing new forms of social interaction, these new artificial agents offer new opportunities to investigate human social cognition ${ }^{2,3}$. In the past decade, the emergence of paradigms comparing Human-Robots Interactions (HRI) to Human-Human Interactions $(\mathrm{HHI})^{2-5}$ opened new avenues for studying social interactions and to better understand how the human social brain, devoted to $\mathrm{HHI}$, adapts to HRI. However, crucial questions remain about our way to engage in HRI or the processes explaining our tendency to attribute mental properties to robots. In the present study, building upon a new $\mathrm{fMRI} \mathrm{HRI/HHI} \mathrm{paradigm,} \mathrm{we} \mathrm{investigate} \mathrm{how} \mathrm{the} \mathrm{human} \mathrm{brain} \mathrm{activity} \mathrm{is} \mathrm{differently}$ affected by repeated interaction with a human or a robot.

To explain human social cognition, the "cognitive systems theory" posits the existence of two exclusive cognitive systems: a "social cognition system" and a "physical cognition system" ${ }^{6-8}$. Numerous neuroimaging studies provide evidence for extended different neural networks specialized in each of 
these systems ${ }^{9,10}$. This distinction was theorized decades ago by philosopher Daniel Dennett, asserting that we adopt an intentional stance (attributing mental states, such as intention, to make sense of an agent's actions, e.g. she takes her car and drive because she wants to go to the restaurant) only when interacting with fellow humans, and a design stance (explaining the agent's actions through functional causation, e.g. the car is propel because of the fuel cells that produce energy chemically) when interacting with mechanical agents because of a lower level of abstraction in explaining an observed action ${ }^{11}$. Results from social cognitive neurosciences latter confirmed that the activation of the social brain was significantly increased when interacting with humans compared to robot or computer agents 12,13 , yet it is possible to adopt, to some extent, an intentional stance toward social robots ${ }^{14,15}$.

However, until now, these investigations mostly consider the interaction as a stable process from beginning to end. In other words, we would adopt a stance at the early stage of an interaction, and would keep it until the end. This assumption contradicts the way humans engage in interactions with others. During an interaction, individuals' perception of the interactor evolves according to the characteristics of the interaction, in particular the representation of the other agent one builds online ${ }^{16,17}$. Therefore, the perception of interactive agents could evolve in time rather than being intrinsically and irremediably dichotomous between physical and social cognition system (i.e. design and intentional stance), a hypothesis never investigated with neurophysiology. For instance, one could adopt an intentional stance as an automatism at first and, through an iterative process, adapt this stance to the interactors (e.g. human, robot). Hence, we posit that depending on the past history at the time of measurement, processes involved in $\mathrm{HHI}$ and HRI could vary differently. These differences could be grounded in a common default level, when the human and robot agents are unfamiliar, that would iteratively specialize according to the type of agent (human vs robot) the individual is facing. We investigated this iterative hypothesis using data from an online HRI/HHI fMRI experiment.

To address the effect of repeated interaction in $\mathrm{HHI}$ and $\mathrm{HRI}$, we performed an analysis of an fMRI study of Human-Human and Human-Robot natural conversations ${ }^{13}$ in order to evaluate whether brain activity remained stable through time or differ according to type of conversant. This issue is fundamental to better understand how we engage in social interactions with anthropomorphic artificial agents and how our brain activity changes through the interaction (compared to interacting with a fellow) ${ }^{18}$.

In the experiment, participants, in an fMRI scanner, discussed alternately with a human agent and a robotic agent. They believed the robot to be autonomous, but it was controlled at distance through a simple Wizard of $\mathrm{Oz}$ interface with a limited number of utterances to select from. They had to discuss images representing "super-heroes" and "rotten fruits" supposedly designed for an advertisement campaign, during twelve trials ( $\approx 1$ minute/trial) for each type of conversant (Fig. 1). This paradigm of online natural discussion comparing human and robot agents is the first of its kind. Overall, from beginning to end, the acquisition of these 24 trials lasted around 40 minutes for each participant.

\section{Materials And Methods}


The present research has been performed in accordance with the Declaration of Helsinki.

\section{Procedure}

The participants $\left(\mathrm{N}=25\right.$ in the corpus (17 women, $\mathrm{M}_{\text {age }}=26.76$ years, $\left.S D .=7.96\right), 21$ included in the analyses) came to the MRI center. The experimenter introduced them to a partner for the experiment (a confederate of the experimenter of the same sex as the participant) and the artificial agent, a backprojected conversational (Furhat) robotic head ${ }^{48}$. Informed consent from participant for publication was obtained.

The gender, voice and various accessories of the agents were adapted to increase the resemblance between the human and the artificial agents. The cover story was presented and the participant was installed in the scanner. Each participant underwent four sessions of approximately eight minutes of functional magnetic resonance imaging (fMRI) acquisition, each consisting of 6 experimental trials that proceeded as follows: a picture appeared for 8,3 seconds, then after a three-second pause with a white fixation cross on a black background, a one-minute live discussion took place with either the human or the artificial agent alternately. Both the participant and interlocutor could hear each other in real-time, and the participant additionally saw a life feed of the interlocutor. In total, each participant took part in twelve one-minute conversations with the human and twelve one-minute conversations with the artificial agent. BOLD signal (Blood Oxygen-Level Dependent) were recorded continuously during each session.

\section{Agent Speech}

The robot is controlled in Wizard of $\mathrm{Oz}$ by an experimenter, the confederate also acting as the human interlocutor: it has a set of pre-recorded answers that the experimenter chooses by pressing virtual buttons on a touch-sensitive tablet. Examples of answers, some generic (e.g. "yes", "no", "maybe", "I don't know", "Can you repeat the question?") and others specific to an image ("It's a yellow pear") or to the campaign ("Maybe it's a campaign to promote local fruit") are given in Table 1.

Table 1. Example of pre-recorded sentences pronounced by the robot, grouped according to their function in the conversation: presentations, generic answers, descriptions of an image (a pear in the series of rotten fruits), and exchanges on the message of the advertising campaign. 
French

Bonjour

Salut

Je m'appelle Furhat

Comment ça va?

Bien

Merci

Oui

Oui c'est possible

Non

Peut-être

C'est une poire

C'est une poire jaune

La poire a l'air malade

Elle paraît faible

Elle semble fatiguée

La poire semble triste

Elle n'a pas l'air contente

Elle semble malheureuse

Peut-être que quelqu'un l'a frappé

Peut-être qu'elle est malade et elle est devenue pourrie

Tu as une idée du message?

C'est peut-être une campagne pour favoriser les fruits locaux

C'est peut-être pour manger des fruits et légumes avant qu'ils pourrissent

Ça pourrait être une pub pour des producteurs de fruits
English

Hello

$\mathrm{Hi}$

My name is Furhat

How's it going?

Good

Thank you

Yes

Yes, it's possible

No

Maybe

It's a pear

It's a yellow pear

The pear looks sick

She seems weak

She looks tired.

The pear looks sad

She doesn't look happy

She seems unhappy

Maybe somebody hit him

Maybe she's sick and she's gone rotten

Do you have any idea what the message is?

Maybe it's a campaign to promote local fruit

Maybe it's to eat fruits and vegetables before they rot

It could be an ad for fruit growers

\section{Data preparation}


The processing of $\mathrm{fMRI}$ data followed standard procedures. The volumes acquired represent the blood oxygenation level-dependent signal (BOLD) in $2.5 \mathrm{~cm}^{3}$ voxels of the brain (repetition time 1.205 seconds). Preprocessing entails a correction of temporal synchronization of the acquired slices, a realignment of the volumes on the first one, and a correction of the deformation due to the local distortions of the magnetic field. Normalization allowed us to put the brains of all participants in the standard MNI space using the DARTEL procedure ${ }^{49}$. Several nuisance covariates were computed to eliminate motion artifacts, potential blood pulse and respiration artifacts, which were highly relevant in a paradigm involving speech, as well as global grey matter signal, white matter activity, and cerebrospinal fluid activity to control global signal fluctuations unrelated to the task (TAPAS toolkit ${ }^{50}$ ).

The analysis of $\mathrm{fMRI}$ data was first based on the general linear model implemented in SPM ${ }^{51}$. Each trial was modelled as a single regressor, and the images presented before each discussion were modelled as a single regressor. We used a brain parcellation formed from functional and connectivity brain data, the Brainnetome atlas ${ }^{19}$, so that regions of interest represented sets of voxels that are homogeneous in terms of function. In each of the 246 regions of the atlas as well as in a hypothalamus mask developed in canonical MNI space ${ }^{52}$ (The hypothalamus is absent from the brainnetome parcellation) the activity estimate was extracted using the MarsBAR toolbox 53 and the set of values for the 21 participants, 12 sessions per agent and 247 regions were used for statistical analyses. The insula corresponded, in Brainnetome parcellation, to regions 163/164-left/right hypergranular insula, posterior, 165/166 left/right ventral agranular insula, anterior, 167/168 left/right agranular insula, anterior, 169/170 left/right ventral dysgranular insula, intermediate, 171/172, dorsal granular insula, intermediate, and 173/174 dorsal dysgranular insula, intermediate. The amygdala corresponded to regions $211 / 212$, left/right medial amygdala, and 213/214, left/right lateral amygdala.

\section{Results}

\section{Analysis strategy}

Here, we investigated where, in the brain, the blood-oxygen-level-dependent (BOLD) signal changes differently in time depending on the nature of the agent. We extracted activity in 246 brain regions of interest, defined by a connectivity-based parcellation of the brain ${ }^{19}$, as well as in a region of interest covering the hypothalamus developed for a previous study ${ }^{13}$. We conducted a whole brain analysis using a multivariate linear mixed model including the 247 Regions Of Interest (to control for repeated test on the same variance and for covariation with other regions), the 12 consecutive trials of interaction (representing time) and the type of agent (human vs. robot). Participants were introduced in the model as a random effect (see method section). The results focus on the interaction between the nature of the agent and the trials in all regions of interest, at the conservative significance threshold of $p<.001$.

\section{Analyses}


Interestingly, we found only five cortical regions for which the activation significantly evolved differently through time for the human and the robot agent. Four of these formed two clusters surrounding the posterior cingulate sulcus bilaterally, as illustrated in Fig. 2. All four regions yielded similar statistical results (Ventral Precuneus Cortex: left: $B=-.14, t(20)=-3.30, \mathrm{Cl}_{95 \%}[-.23 ;-.06]$; right: $B=-.15, t(20)=$ $-3.48, \mathrm{Cl}_{95 \%}[-.23 ;-.06]$; Posterior Cingulate Cortex[BA23d]: $B=-.16, t(20)=-3.82, \mathrm{Cl}_{95 \%}[-.24 ;-.08]$; right: $B=-.15, t(20)=-3.67, \mathrm{Cl}_{95 \%}[-.24 ;-.07]$; all $\left.p s<.001\right)$. The pattern of results was also the same in the four areas (Fig. 3), an activity increasing with time in $\mathrm{HHI}$ (Ventral Precuneus Cortex: left: $B=.11, t(20)=4.01$, $\mathrm{Cl}_{95 \%}$ [.06; .18]; right: $B=.10, t(20)=3.45, \mathrm{Cl}_{95 \%}[.04 ; .16]$; Posterior Cingulate Cortex: left: $B=.12, t(20)=$ 4.09, $\mathrm{Cl}_{95 \%}$ [.06; .18]; right: $B=.11, t(20)=3.83, \mathrm{Cl}_{95 \%}[.05 ; .17]$; all $\left.p \mathrm{~s}<.001\right)$, with no effect of time in HRI (Ventral Precuneus Cortex: left: $B=-.02, t(20)=-.52, \mathrm{Cl}_{95 \%}[-.07 ; .04]$; right: $B=-.04, t(20)=-1.36, \mathrm{Cl}_{95 \%}$ [-.10; .02]; Posterior Cingulate Cortex left: $B=-.04, t(20)=-1.30, \mathrm{Cl}_{95 \%}[-.10 ; .02]$; right: $B=-.04, t(20)=$ $-1.32, \mathrm{Cl}_{95 \%}[-.09 ; .02]$; all $\left.p s>.001\right)$. The brain activity increases bilaterally with time in these posterior medial regions of interest only when interacting with the human. Interestingly, none of these regions were significantly affected by the nature of the interacting agent without taking into account the time variable. In other words, without considering the effect of time on the BOLD signal, one would have concluded that activity in this core area of the social brain shows no difference between $\mathrm{HHI}$ and HRI.

A significant interaction between the nature of the agent and the trials was found in a region of interest corresponding to the right fusiform gyrus $\left(B=-.14, t(20)=-3.64, \mathrm{Cl}_{95 \%}[-.22 ;-.07], p<.001\right)$. The pattern was different from regions of the previous cluster, as the activity didn't significantly changed in $\mathrm{HHI}(B=$ $\left.-.02, t(20)=.73, \mathrm{Cl}_{95 \%}[-.03 ; .07], p=.466\right)$, but decreased with time in $\mathrm{HRI}\left(B=-.12, t(20)=-4.25, \mathrm{Cl}_{95 \%}\right.$ $[-.17 ;-.06], p<.001)$. Given that this area is isolated, that the fusiform gyrus activity is mainly related to visual processing, and that response to the robot, but not the human, changed with time, one possible interpretation is the habituation to the novelty represented by the conversational robot's face ${ }^{20,21}$, which is of limited interest given our theoretical question. Therefore, we will not further discuss the response of this region.

\section{Discussion}

The present analysis identifies an involvement of the Posterior Cingulate Cortex (PCC) in a dynamic aspect of natural social interactions. This region broadly corresponds to Brodmann area 31 and Brodmann area 23. Across primates species including humans ${ }^{22}$, these isocortical areas of the PCC, rather difficult to differentiate by cytoarchitectonics ${ }^{23}$, are characterized by an increase of size and proportion of superficial layers interpreted as "a progressive structural differentiation permitting an increasing amount of intracortical information-processing capacities" ${ }^{23}$. Involvement of the PCC has been reported in a number of tasks pertaining to social cognition ${ }^{22}$, such as self-reflection ${ }^{22}$, the sense of agency (attributing executed or imagined actions to the other or to oneself, ${ }^{24,25}$ respectively), familiarity (of people ${ }^{26}$, of places and objects ${ }^{27}$ ), or emotional judgements (of words ${ }^{28}$ or faces ${ }^{29}$ ). The PCC activates across nearly all tasks that require self-generated thoughts, including self-referential 
processing, episodic or autobiographical memory, future thinking, mentalizing, spatial navigation, and conceptual processing ${ }^{30-32}$. One common theme could be to associate memory and emotional processing, given it is part of the limbic system directly connected to parahippocampal areas ${ }^{33}$. It is close and connected to early visual areas, in particular the ventral stream, a possible explanation of its involvement in mental imagery ${ }^{22,24}$. The PCC is also the central node of the Default Mode Network, defined by an increased connectivity during rest compared to task-oriented activity ${ }^{34,35}$. It is now well established that the DMN and the social brain areas overlap largely ${ }^{36}$, one hypothesis being that the default mode network corresponds to situations in which the brain is free to monitor both the environment and inner world without task constraints ${ }^{22}$. Altogether, it was proposed that the PCC plays a central role in adapting behaviors to changes in the world: it acts as a gateway controlling whether and which task-positive network should be engaged as a function of external information on the one hand, and self-episodic memory and emotional processing ${ }^{37}$ on the other hand.

Current results further the understanding of the involvement of this region by showing its activity is evolving during natural social interactions. These changes argue for a dynamic process in $\mathrm{HHI}$ with an increase of social engagement with the human agent as an iterative process. In contrast, in HRI, this process remains stable in time. While it is not possible to ascertain the origin of these different dynamics, the fact that responses to human and robot are first undistinguishable before becoming significantly different argues in favor of the accumulation of social information during repeated interactions with the human, while this robot provides limited social signals. These different dynamics in the PCC could represent the building of familiarity or trust and a deepening of the (emotional) engagement in the interaction restricted to the human interlocutor, even within a short period of time. This result echoes the top-down control postulated by Daniel Denett's intentional stance that postulates that we would rather adopt a design stance when facing a machine ${ }^{38}$. As such we could expect a drop of PCC activity on the long-term in HRI as theorized in the initialization, familiarization and stabilization framework ${ }^{39}$. In this view the common default level could be explained by the novelty effect during the initialization stage ${ }^{40}$, the lack of knowledge to develop a target-specific representation about the robot ${ }^{41}$, the will to efficiently interact with the robot (and other motivational factors) ${ }^{42}$ and as a consequence, the tendency to mimic the way we engage with humans on HRI ${ }^{43}$. This short-term stage would be followed by a drop of the mentalistic attributions (intentional stance) because the initial apparent complexity of the robot vanishes to finally stabilize.

Therefore, one question needs to be asked: "could we countervail this drop?". Some authors posit that reaching a certain threshold of social complexity, robots could maintain users' social engagement ${ }^{44}$. For instance, adding some non-monotonic (evolving through time) and/or social behaviour such as selfdisclosure ${ }^{45}$, non-verbal behaviours such as eye contact, joint attention and emotions ${ }^{46}$ or social support ${ }^{44,47}$ could help to maintain the intentional stance on a longer period.

Only the brain regions around the posterior cingulate sulcus was found to evolve differently in time between the natural and the artificial agent, and this region is at the core of the social brain network. 
Interestingly, response to both agents, indistinguishable at first, increase significantly through time with the human partner leading to a significant difference in only twelve minutes of interactions with both human and robot agents. Indeed, interaction with humans (compared to robots) triggers an iterative bidirectional social cognition processing. Our results illustrate that when engaging in a social interaction with unknown agents, one's level of social engagement is updated through the interaction (short-term), according to the other agent's feedback. The iterative increase of the social processing during interaction showed in $\mathrm{HHI}$ but not in HRI is fundamental for social engagement. Hence, a crucial question arises for HRI: Could robots be considered viable social agents after the initial encounter? Presently, the results argue for a negative answer. However, the present paradigm offers an opportunity to directly assess how the designs of robots and emergence of social cues could influence human social brain activity comparing online $\mathrm{HHI}$ and HRI as a new form of Turing test. Finally, as a core message for HRI research, the crucial question should not be focused merely on whether humans activate their default social cognition system interacting with robots, but also whether and how, similarly to HHI, HRI may trigger an increased activity of the social cognition system in humans.

\section{Declarations}

Author Contributions: TC collected the data, NS analyzed the data, NS and TC drafted the manuscript

Competing Interest Statement: Authors declare no competing interest

Classification: Social Sciences · Psychological and Cognitive Sciences

\section{Acknowledgments}

\section{Data accessibility}

Transcribed linguistic data can be found on Ortolang (https://www.ortolang.fr): https://hdl.handle.net/11403/convers; fMRI group data on Neurovault (https://neurovault.org/): /collections/ASGXRWEM/; fMRI raw data can be found on OpenNeuro (https://openneuro.org/): https://openneuro.org/datasets/ds001740.

\section{Fundings}

Research supported by grants ANR-16-CONV-0002 (ILCB), ANR-11-LABX-0036 (BLRI) and AAP-ID-17-46170301-11.1 by the Excellence Initiative of Aix-Marseille University ( $A *$ MIDEX), a French "Investissement d'Avenir" programme. B.R. is supported by the Fondation pour la Recherche Médicale (FRM, SPF20171039127).

\section{Ethics}

The project received ethical approval from the Comité de Protection des Personnes (CPP) Sud-Marseille 1 (approval number 2016-A01008-43). Written consent was obtained from all participants. 
Informed consent was obtained from all subjects.

\section{References}

1. Mithen, S. \& Boyer, P. Anthropomorphism and the Evolution of Cognition. J. R. Anthropol. Inst.2, 717 (1996).

2. Wiese, E., Metta, G. \& Wykowska, A. Robots as intentional agents: Using neuroscientific methods to make robots appear more social. Frontiers in Psychology vol. 8 (2017).

3. Wykowska, A., Chaminade, T. \& Cheng, G. Embodied artificial agents for understanding human social cognition. Philosophical Transactions of the Royal Society B: Biological Sciences vol. 371 (2016).

4. Schilbach, L. A second-person approach to other minds. Nat. Rev. Neurosci.11, 449 (2010).

5. Chaminade, T. An experimental approach to study the physiology of natural social interactions. Interact. Stud. Soc. Behav. Commun. Biol. Artif. Syst. Stud. / Soc. Behav. Commun. Biol. Artif. Syst. Stud.18, 254-275 (2017).

6. Jack, A. I. et al. FMRI reveals reciprocal inhibition between social and physical cognitive domains. Neuroimage66, 385-401 (2013).

7. Evans, J. S. B. T. Dual-processing accounts of reasoning, judgment, and social cognition. Annu. Rev. Psychol.59, 255-278 (2008).

8. Evans, J. S. B. T. \& Stanovich, K. E. Dual-Process Theories of Higher Cognition: Advancing the Debate. Perspect. Psychol. Sci.8, 223-241 (2013).

9. Jack, A. I., Dawson, A. J. \& Norr, M. E. Seeing human: Distinct and overlapping neural signatures associated with two forms of dehumanization. Neuroimage79, 313-328 (2013).

10. Martin, A. \& Weisberg, J. Neural foundations for understanding social and mechanical concepts. Cognitive Neuropsychology vol. 20 575-587 (2003).

11. Dennett, D. C. Intentional Systems. J. Philos.68, 87-106 (1971).

12. Cheung, J. C.-S. Alone Together: Why We Expect More from Technology and Less from Each Other. Journal of Social Work Practice vol. 27 (Basic Books, 2013).

13. Rauchbauer, B. et al. Brain activity during reciprocal social interaction investigated using conversational robots as control condition. Philos. Trans. R. Soc. B Biol. Sci.374, (2019).

14. Abu-Akel, A. M., Apperly, I. A., Wood, S. J. \& Hansen, P. C. Re-imaging the intentional stance. Proc. R. Soc. B Biol. Sci.287, 20200244 (2020). 
15. Perez-Osorio, J. \& Wykowska, A. Adopting the intentional stance toward natural and artificial agents. Philos. Psychol.33, 369-395 (2020).

16. Butler, E. A. Emotions are temporal interpersonal systems. Current Opinion in Psychology (2017) doi:10.1016/j.copsyc.2017.07.005.

17. Butler, E. A. Temporal interpersonal emotion systems: The 'TIES' that form relationships. Personality and Social Psychology Review (2011) doi:10.1177/1088868311411164.

18. Yang, G. Z. et al. The grand challenges of science robotics. Sci. Robot.3, eaar7650 (2018).

19. Fan, L. et al. The Human Brainnetome Atlas: A New Brain Atlas Based on Connectional Architecture. Cereb. Cortex26, 3508-3526 (2016).

20. Thompson, R. F. Habituation: A history. Neurobiol. Learn. Mem.92, 127-134 (2009).

21. Rankin, C. H. et al. Habituation revisited: An updated and revised description of the behavioral characteristics of habituation. Neurobiol. Learn. Mem.92, 135-138 (2009).

22. Vogt, B. A., Vogt, L. \& Laureys, S. Cytology and functionally correlated circuits of human posterior cingulate areas. Neuroimage29, 452-466 (2006).

23. Zilles, K., Armstrong, E., Schlaug, G. \& Schleicher, A. Quantitative cytoarchitectonics of the posterior cingulate cortex in primates. J. Comp. Neurol.253, 514-524 (1986).

24. Farrer, C. \& Frith, C. D. Experiencing oneself vs another person as being the cause of an action: The neural correlates of the experience of agency. Neuroimage15, 596-603 (2002).

25. Ruby, P. \& Decety, J. Effect of subjective perspective taking during simulation of action: A PET investigation of agency. Nat. Neurosci.4, 546-550 (2001).

26. Maddock, R. J., Garrett, A. S. \& Buonocore, M. H. Remembering familiar people: The posterior cingulate cortex and autobiographical memory retrieval. Neuroscience104, 667-676 (2001).

27. Sugiura, M., Shah, N. J., Zilles, K. \& Fink, G. R. Cortical representations of personally familiar objects and places: Functional organization of the human posterior cingulate cortex. J. Cogn. Neurosci.17, 183198 (2005).

28. Maddock, R. J., Garrett, A. S. \& Buonocore, M. H. Posterior cingulate cortex activation by emotional words: fMRI evidence from a valence decision task. Hum. Brain Mapp.18, 30-41 (2003).

29. Fusar-Poli, P. et al. Laterality effect on emotional faces processing: ALE meta-analysis of evidence. Neurosci. Lett.452, 262-267 (2009). 
30. Brewer, J. A., Garrison, K. A. \& Whitfield-Gabrieli, S. What about the 'self' is processed in the posterior cingulate cortex? Front. Hum. Neurosci. (2013) doi:10.3389/fnhum.2013.00647.

31. Qin, P. \& Northoff, G. How is our self related to midline regions and the default-mode network? Neurolmage vol. 57 1221-1233 (2011).

32. Spreng, R. N., Mar, R. A. \& Kim, A. S. N. The common neural basis of autobiographical memory, prospection, navigation, theory of mind, and the default mode: A quantitative meta-analysis. J. Cogn. Neurosci.21, 489-510 (2009).

33. Vogt, B. A., Finch, D. M. \& Olson, C. R. Functional heterogeneity in cingulate cortex: The anterior executive and posterior evaluative regions. Cereb. Cortex2, 435-443 (1992).

34. Wang, R. W. Y., Chang, W. L., Chuang, S. W. \& Liu, I. N. Posterior cingulate cortex can be a regulatory modulator of the default mode network in task-negative state. Sci. Rep.9, (2019).

35. Fransson, P. \& Marrelec, G. The precuneus/posterior cingulate cortex plays a pivotal role in the default mode network: Evidence from a partial correlation network analysis. Neuroimage42, 1178-1184 (2008).

36. Mars, R. B. et al. On the relationship between the 'default mode network' and the 'social brain'. Front. Hum. Neurosci.6, 1-9 (2012).

37. Leech, R. \& Sharp, D. J. The role of the posterior cingulate cortex in cognition and disease. Brain vol. $13712-32$ (2014).

38. Dennett, D. C. Intentional Systems. J. Philos.68, 87-106 (1971).

39. Lemaignan, S., Fink, J. \& Dillenbourg, P. The dynamics of anthropomorphism in robotics. in ACM/IEEE International Conference on Human-Robot Interaction 226-227 (2014). doi:10.1145/2559636.2559814.

40. Leite, I., Martinho, C., Pereira, A. \& Paiva, A. As time goes by: Long-term evaluation of social presence in robotic companions. in Proceedings - IEEE International Workshop on Robot and Human Interactive Communication 669-674 (2009). doi:10.1109/ROMAN.2009.5326256.

41. Epley, N., Waytz, A. \& Cacioppo, J. T. On Seeing Human: A Three-Factor Theory of Anthropomorphism. Psychol. Rev.114, 864-886 (2007).

42. Epley, N., Waytz, A., Akalis, S. \& Cacioppo, J. T. When we need a human: Motivational determinants of anthropomorphism. Soc. Cogn.26, 143-155 (2008).

43. Urquiza-Haas, E. G. \& Kotrschal, K. The mind behind anthropomorphic thinking: Attribution of mental states to other species. Anim. Behav.109, 167-176 (2015).

44. Leite, I. et al. The influence of empathy in human-robot relations. Int. J. Hum. Comput. Stud.71, 250260 (2013).

Page $12 / 14$ 
45. Tanaka, F., Cicourel, A. \& Movellan, J. R. Socialization between toddlers and robots at an early childhood education center. Proc. Natl. Acad. Sci. U. S. A.104, 17954-17958 (2007).

46. Kozima, H., Michalowski, M. P. \& Nakagawa, C. Keepon: A playful robot for research, therapy, and entertainment. Int. J. Soc. Robot.1, 3-18 (2009).

47. Kirby, R., Forlizzi, J. \& Simmons, R. Affective social robots. Rob. Auton. Syst.58, 322-332 (2010).

48. Al Moubayed, S., Beskow, J., Skantze, G. \& Granström, B. Furhat: A back-projected human-like robot head for multiparty human-machine interaction. in Lecture Notes in Computer Science (including subseries Lecture Notes in Artificial Intelligence and Lecture Notes in Bioinformatics) vol. 7403 LNCS 114-130 (2012).

49. Ashburner, J. A fast diffeomorphic image registration algorithm. Neuroimage38, 95-113 (2007).

50. Kasper, L. et al. The PhysIO Toolbox for Modeling Physiological Noise in fMRI Data. J. Neurosci. Methods276, 56-72 (2017).

51. Penny, W., Friston, K., Ashburner, J., Kiebel, S. \& Nichols, T. Statistical Parametric Mapping: The Analysis of Functional Brain Images. Statistical Parametric Mapping: The Analysis of Functional Brain Images (2007). doi:10.1016/B978-0-12-372560-8.X5000-1.

52. Wolfe, F. H., Auzias, G., Deruelle, C. \& Chaminade, T. Focal atrophy of the hypothalamus associated with third ventricle enlargement in autism spectrum disorder. Neuroreport26, 1017-1022 (2015).

53. Brett, M., Anton, J. L., Valabregue, R. \& Poline, J. B. Region of interest analysis using an SPM toolbox. in 8th international conference on functional mapping of the human brain vol. 16497 (2002).

\section{Figures}
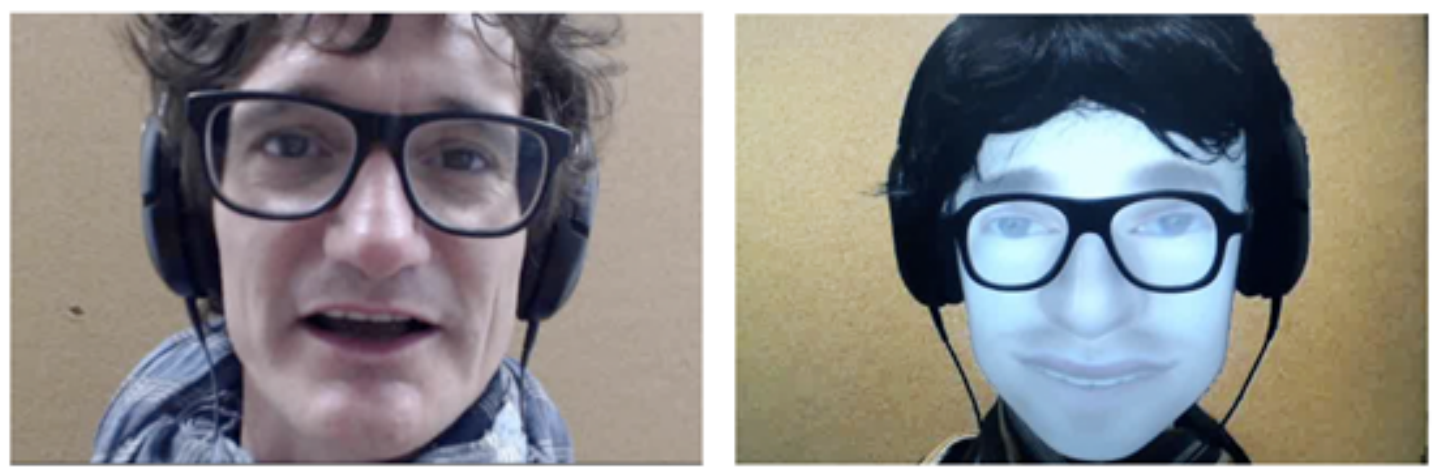

\section{Figure 1}

Single frames extracted from the live video feeds projected on a screen in front of the scanned participants' eyes, with the Human (experimenter) (left) vs Robot (right) male conversant. 


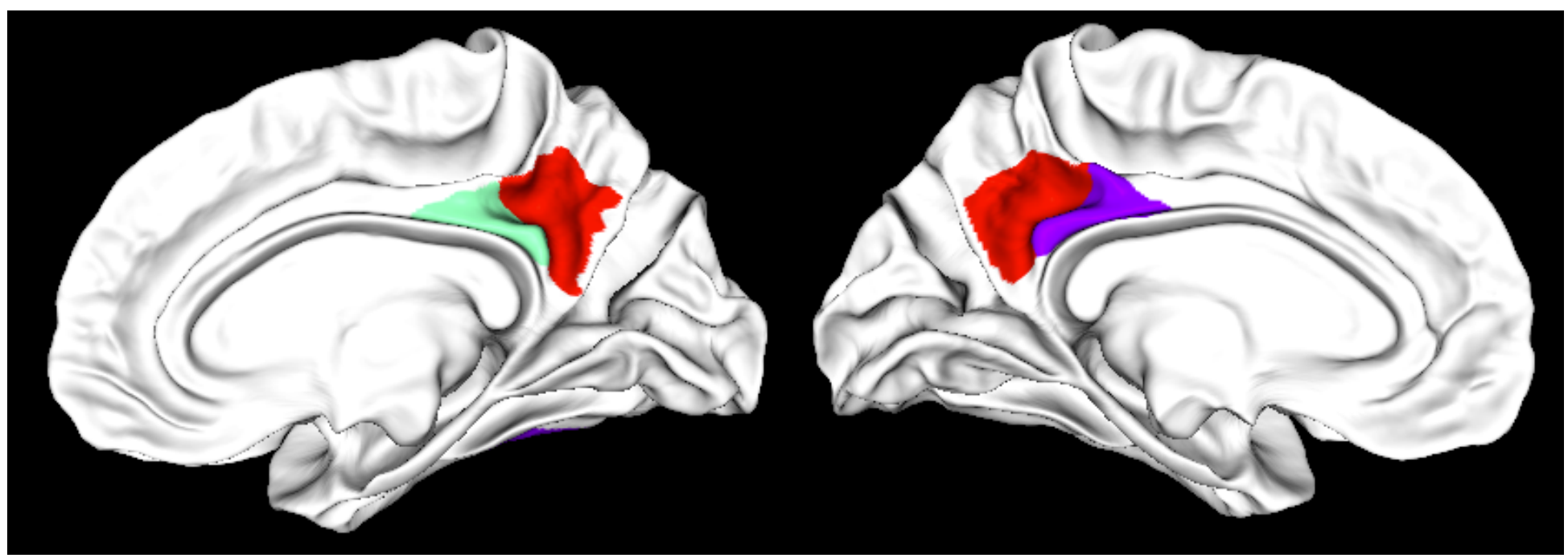

Figure 2

Regions of interest in the posterior cingulate and precuneus (red) where the bold signal is differently affected by time depending on the nature of the agent.

Left

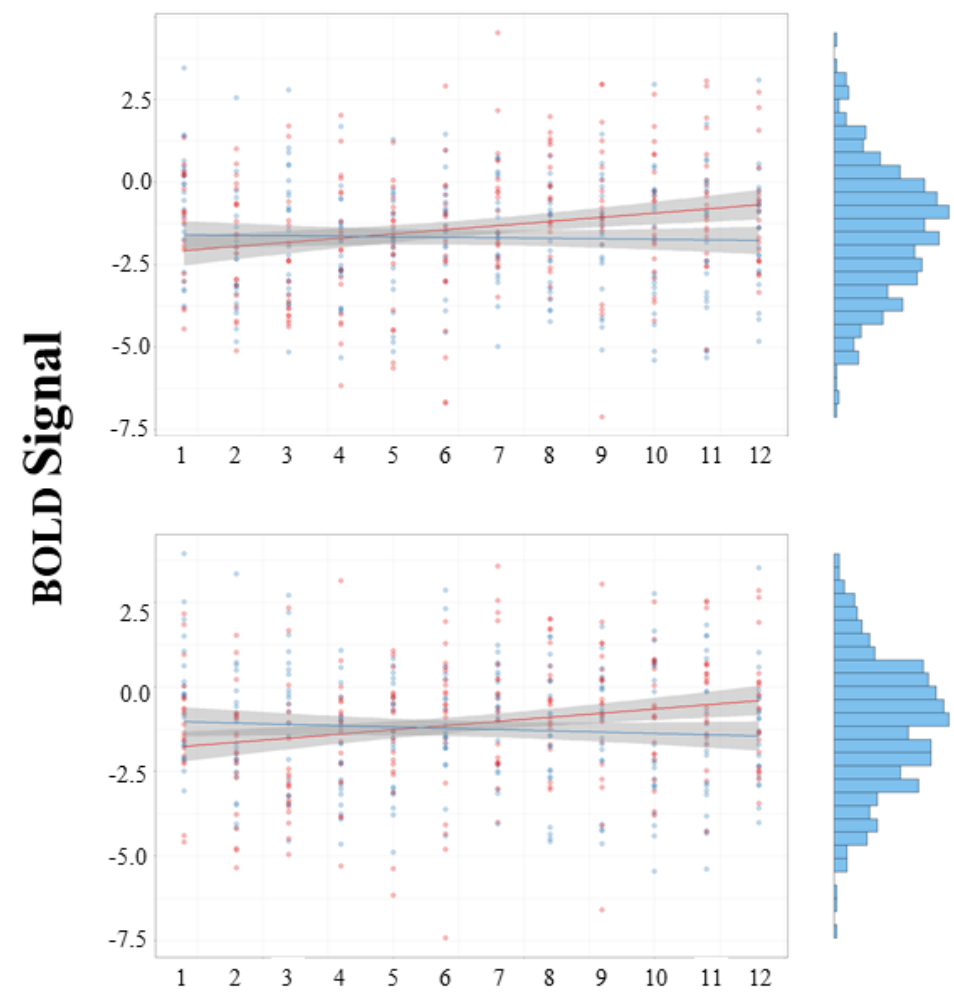

Trials
Right
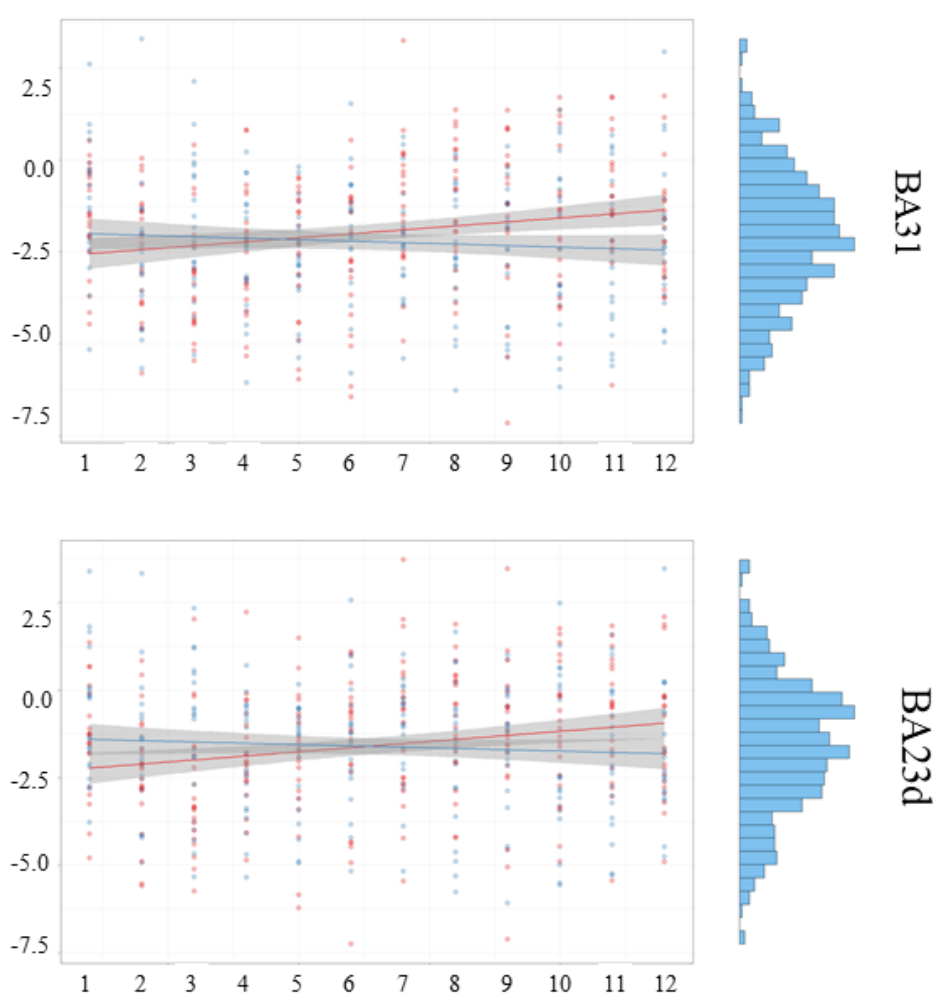

\section{Figure 3}

BOLD signal as a function of the type of interactor (human vs robot) and time (12 trials) in Brodmann areas 31 (upper) and 23d (lower) bilaterally. 